\title{
Tailoring re-entrant geometry in inverse colloidal monolayers to control surface wettability
}

\section{Citation}

Utech, Stefanie, Karina Bley, Joanna Aizenberg, and Nicolas Vogel. 2016. “Tailoring Re-Entrant Geometry in Inverse Colloidal Monolayers to Control Surface Wettability." Journal of Materials Chemistry A 4 (18): 6853-6859. doi:10.1039/c5ta08992a.

\section{Published Version}

doi:10.1039/C5TA08992A

\section{Permanent link}

http://nrs.harvard.edu/urn-3:HUL.InstRepos:37253516

\section{Terms of Use}

This article was downloaded from Harvard University's DASH repository, and is made available under the terms and conditions applicable to Open Access Policy Articles, as set forth at http:// nrs.harvard.edu/urn-3:HUL.InstRepos:dash.current.terms-of-use\#OAP

\section{Share Your Story}

The Harvard community has made this article openly available.

Please share how this access benefits you. Submit a story.

\section{Accessibility}


Tailoring re-entrant geometry in inverse colloidal monolayers to control surface wettability Stefanie Utech ${ }^{1}$, Karina Bley ${ }^{1}$, Joanna Aizenberg ${ }^{2}$ and Nicolas Vogel ${ }^{1,3 *}$

\author{
${ }^{1}$ Institute of Particle Technology, Friedrich-Alexander University Erlangen-Nürnberg, Haberstr. 9a, 91058 \\ Erlangen, Germany \\ ${ }^{2}$ John A. Paulson School of Engineering and Applied Sciences, Harvard University, 9 Oxford Street, \\ Cambridge, MA 02139, USA \\ ${ }^{3}$ Cluster of Excellence Engineering of Advanced Materials, Friedrich-Alexander University Erlangen- \\ Nürnberg, Nägelsbacherstr. 49, 91054 Erlangen, Germany
}

\begin{abstract}
Controlling the microscopic wetting state of a liquid in contact with a structured surface is the basis for the design of liquid repellent as well as anti-fogging coatings by preventing or enabling a given liquid to infiltrate the surface structures. Similarly, a liquid can be confined to designated surface areas by locally controlling the wetting state, with applications ranging from liquid transport on a surface to creating tailored microenvironments for cell culture or chemical synthesis. The control of the wetting of a lowsurface-tension liquid is substantially more difficult compared to water and requires surface structures with overhanging features, known as re-entrant geometries.

Here, we use colloidal self-assembly and templating to create two-dimensional nanopore arrays with tailored re-entrant geometry. These pore arrays, termed inverse monolayers, are prepared by backfilling a sacrificial colloidal monolayer with a silica sol-gel precursor material. Varying the precursor concentration enables us to control the degree to which the colloids are embedded into the silica matrix. Upon calcination, nanopores with different opening angles result. The pore opening angle directly correlates with the re-entrant curvature of the surface nanostructures and can be used to control the macroscopic wetting behavior of a liquid sitting on the surface structures. We characterize the wetting of various liquids by static and dynamic contact angles and find correlation between the experimental results and theoretical predictions of the wetting state based on simple geometric considerations. We demonstrate the creation of omniphobic surface coatings that support Cassie-Baxter wetting states for liquids with low surface tensions, including octane $(\gamma=21.7 \mathrm{mN} / \mathrm{m})$. We further use photolithography to spatially confine such low-surface-tension liquids to desired areas of the substrate with high accuracy.
\end{abstract}




\section{Introduction}

Colloidal particles can be self-assembled into ordered superstructures in two and three dimensions, giving access to materials with defined periodicities at the nanoscale by a simple bottom-up process. ${ }^{1-3}$ Twodimensional colloidal crystals, commonly known as colloidal monolayers, are especially attractive as templates and masks to create large-area surface patterns with nanoscale dimensions by successive material deposition or removal steps. ${ }^{4-6}$ Such surface coatings find applications in structural coloration, ${ }^{7-8}$ light management in solar cells, ${ }^{9}$ anti-reflective coatings, ${ }^{10-12}$ or to impart self-cleaning and superhydrophobic properties. ${ }^{13-17}$

Superhydrophobic surfaces typically mimic nature's famously self-cleaning lotus leaves. ${ }^{18}$ Their repellent properties are based on the creation of a composite air/solid interface by introducing topographic features at the micro- and nanoscale to a surface bearing a chemical functionality that maximizes the contact angle with the liquid to be repelled. ${ }^{19-22}$ Liquids with high surface tensions, predominantly water, can easily be prevented from infiltrating the roughness features; resting on a cushion of air and being in contact only with the tips of the structures, a situation that is known as the Cassie-Baxter state. ${ }^{23}$ Superhydrophobic surfaces with efficient water shedding and self-cleaning properties result. ${ }^{24-25}$ However, the design of surfaces that also repel low-surface-tension liquids such as oils is significantly more challenging. The challenge arises from the fact that regular surface features with vertical side walls only support a CassieBaxter wetting state with liquids having a contact angle of $>90^{\circ}$ on a flat surface. Even the most repellent surface functionalities (typically featuring long perfluorinated alkyl chains) do not support sufficiently high contact angles for low-surface-tension liquids such as hydrocarbon oils. Hence, these liquids will not be expelled from most surface features but infiltrate the structure and form a Wenzel wetting state. ${ }^{26}$ It has been recognized that the design of an oleophobic coating requires the presence of re-entrant curvature, i.e. surface topography features that bend towards the substrate and form angles with the substrates below $90^{\circ} .{ }^{27-29}$ If this angle is lower than the contact angle of the applied liquid, a convex meniscus shape results that pushes the liquid away from the sidewalls of the surface features and thus enables the creation of a metastable Cassie-Baxter state for low-surface-tension liquids. ${ }^{27-32}$

Here, we use colloidal templating to prepare two-dimensional nanopore arrays known as inverse colloidal monolayers ${ }^{33-35}$ as surface coatings that provide precisely adjustable re-entrant curvature without relying on complex fabrication protocols or hierarchical structure design. This simple nanoscale architecture enables us to rationally tailor the macroscopic wetting state, even for low-surface-tension liquids. The inverse monolayers are prepared by backfilling a colloidal monolayer with a sol-gel precursor. Upon calcination, the colloidal particles are combusted, creating arrays of nanopores with size and order 
reflecting the dimensions and quality of the colloidal monolayer. The inverse geometry provides several advantages over normal colloidal monolayers. First, the constituent material typically consists of oxides formed from the sol-gel process, which enables simple and versatile surface functionalization using silane chemistry. Second, the close-cell structure provides mechanical robustness since all features are interconnected and covalently bond to the substrate, rendering them much more resistant to abrasion and mechanical damage. ${ }^{35}$ Finally, the inversion of the convex surface structure of the colloids provides concave surface features with overhang, ${ }^{36}$ a prerequisite for controlling wettability of the surface features.

We prepare inverse colloidal monolayers over macroscopic areas by spin-coating of a silica sol-gel precursor solution onto a pre-assembled colloidal monolayer following an established protocol. ${ }^{35}$ In brief, a colloidal monolayer is assembled at the air/water interface and manually transferred onto a solid substrate. $^{37} \mathrm{~A}$ mixture of tetraethylorthosilicate (TEOS), ethanol and hydrochlorid acid (0.1N) is hydrolyzed for one hour, added to the monolayer surface and spin-coated to solidify. After calcination to burn out the template colloids, the inverse monolayer architecture results (Figure 1a). By controlling the concentration of the sol-gel precursor, we can tailor the pore opening: The template colloids are embedded more and more into the silica thin-film with increasing amount of added solid material (Figure 1b). The pore opening angle $\Psi$ is the crucial parameter that determines the re-entrant geometry of the structures and is directly related to the diameter of the pore opening $a$ and the pore diameter $d$ (Figure 1c):

$$
\sin (\Psi)=a / d
$$

Increasing the sol-gel concentration thus enables us to create surface structures with tailored re-entrant curvature. Figure 1e shows examples of structures with a pore opening angle ranging from $79^{\circ}$ to $23^{\circ}$. Table 1 provides details of the structural parameters and preparation protocols. The high regularity and uniformity of these self-assembled surface structures is exemplarily shown in a low resolution SEM image in Figure 1d. We chose colloids with a diameter of $1060 \mathrm{~nm}$ for the experiments since these enabled us to prepare inverse monolayer with a wide range of opening angles needed to establish their wetting properties in detail. While the methodology can be generally applied to smaller particle sizes, ${ }^{35}$ adjustment of the opening angle becomes increasingly difficult due to the increasingly smaller differences in the silica film thickness required for different opening angles. 

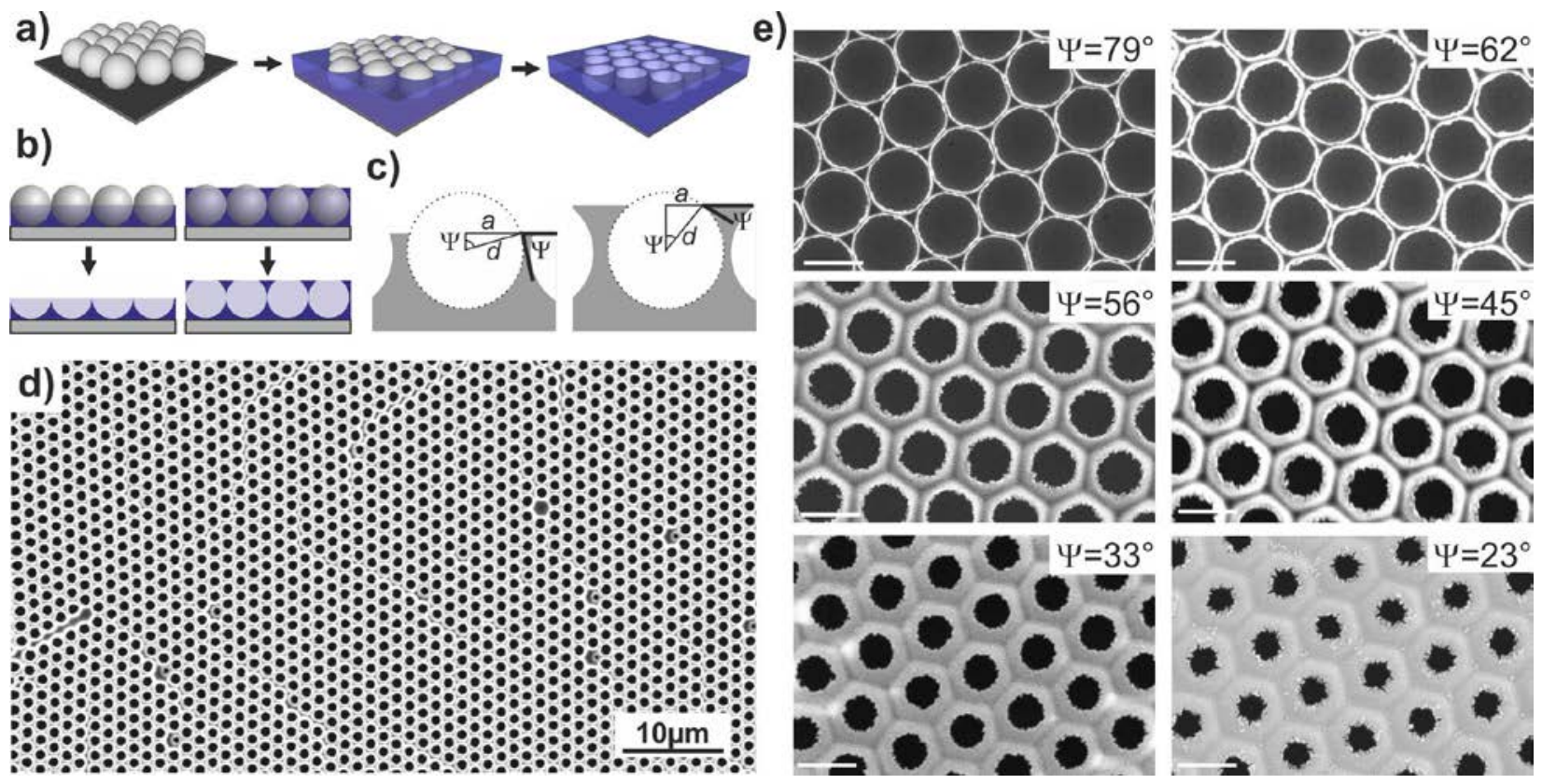

Figure 1. Fabrication of re-entrant surface structures from colloidal monolayer templates. a) Schematic

illustration of the fabrication process. A monolayer of polymer colloids is deposited onto a solid substrate, backfilled with a silica precursor and calcined to create an inverse replica of the monolayer. b) The pore opening can be precisely controlled by varying the amount of silica precursor applied. c) Geometric illustration of the relationship between the pore opening $a$, colloidal radius $d$ and the pore opening angle $\Psi$ for two exemplary pore openings. d) Low magnification SEM image demonstrating the high degree of order and uniformity of the prepared nanopore arrays. e) SEM images of inverse monolayer with different pore openings demonstrating control of the re-entrant geometry of the surface structures of the inverse monolayer structures. All scale bars are $1 \mu \mathrm{m}$. 
Table 1. Structural parameters and details of preparation for inverse monolayer structures with different degrees of re-entrant curvature. All samples were prepared from 1060nm polystyrene colloids as templates.

\begin{tabular}{|c|c|c|c|c|}
\hline $\begin{array}{l}\text { Pore opening } \\
\qquad a / \mathrm{nm}\end{array}$ & $\begin{array}{c}\text { Pore opening angle } \\
\qquad \Psi{ }^{\circ}\end{array}$ & $\begin{array}{l}\text { Solid fraction* } \\
\qquad \Phi\end{array}$ & Sol-gel mixture** & $\begin{array}{c}\text { Dilution with } \\
\text { Ethanol } \\
\text { (TEOS } \\
\text { mixture:ethanol) }\end{array}$ \\
\hline $1040 \pm 30$ & 79 & 0.13 & $\mathrm{~A}$ & $1: 0.9$ \\
\hline $940 \pm 29$ & 62 & 0.29 & B & $1: 2$ \\
\hline $880 \pm 21$ & 56 & 0.38 & A & 1:0.7 \\
\hline $846 \pm 25$ & 53 & 0.43 & A & 1:0.6 \\
\hline $742 \pm 19$ & 45 & 0.55 & A & 1:0.5 \\
\hline $570 \pm 23$ & 33 & 0.74 & B & $1: 1.3$ \\
\hline $428 \pm 26$ & 23 & 0.86 & B & 1:1.1 \\
\hline
\end{tabular}

* The area of a normal projection of the surface covered by solid material (instead of pores).

**A) $1: 1.5: 1$ mixture of $\mathrm{HCl} 0.1 \mathrm{~N}:$ Ethanol : TEOS by weight. B) $1: 1.5: 2 \mathrm{HCl} 0.1 \mathrm{~N}:$ Ethanol : TEOS by weight.

We use silane chemistry to functionalize the surface of the inverse monolayer structures with $(1 \mathrm{H}, 1 \mathrm{H}, 2 \mathrm{H}, 2 \mathrm{H}$-tridecafluorooctyl)-trichlorosilane to maximize the contact angle formed with both water and hydrocarbon oils. Figure 2 shows the apparent omniphobic behavior of the inverse monolayer structures with a pore opening of $79^{\circ}$. The contact angle of liquids with surface tension ranging from 21.7 $\mathrm{mN} / \mathrm{m}$ (octane) to $72.8 \mathrm{mN} / \mathrm{m}$ (water) on the inverse monolayer structures strongly increases compared to a flat reference surface with similar surface chemistry and shows values well above $100^{\circ}$ for all tested liquids (Figure 2). 


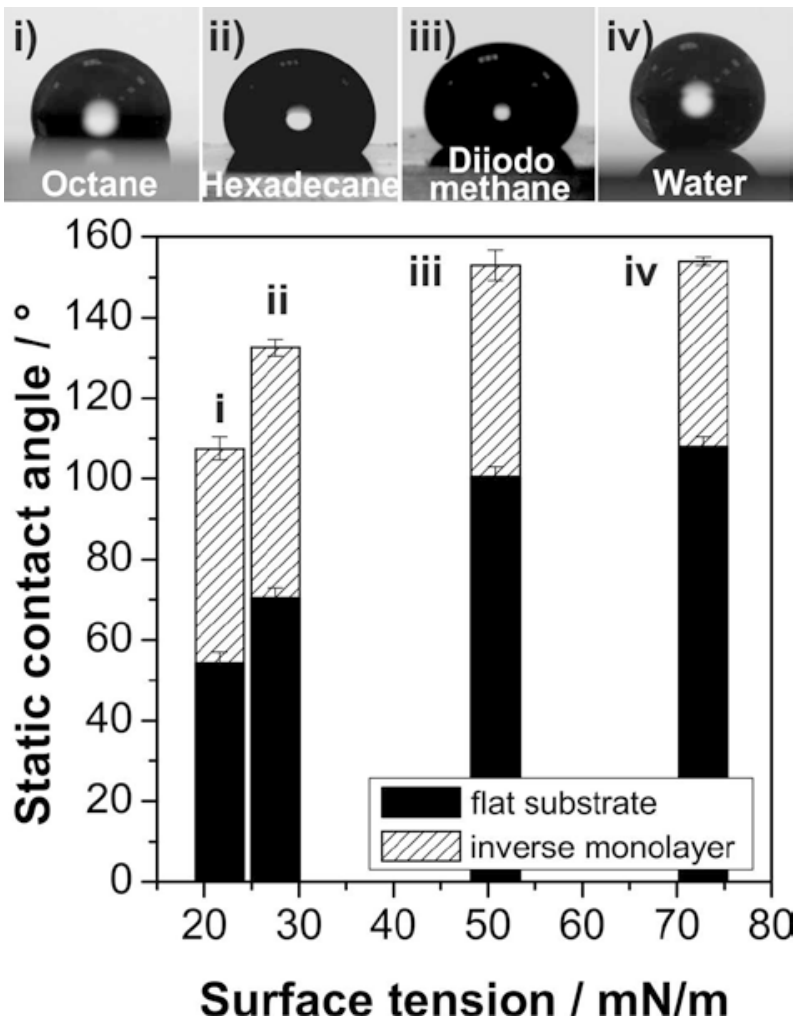

Figure 2. Liquids with various surface tensions on inverse monolayers functionalized with fluorinated surface chemistry form contact angles well above $100^{\circ}$ (pore opening angle of the inverse monolayer $79^{\circ}$ ).

However, the high static contact angle the liquids form with the substrate does not directly describe the wetting state of the liquid in contact with the pores of the inverse monolayers. For most practical purposes, for example facilitating the shedding a liquid droplet, liquid separation or confining a desired liquid to a designated area on the surface, it is crucial to gain control of the wetting state; i.e. to control whether or not a given liquid infiltrates the pores. From geometric considerations illustrated in Figure 3a, pore infiltration is expected when the contact angle the liquid forms with a flat surface of identical surface functionality, $\Theta$, is smaller than the angle of the re-entrant surface structure of the inverse monolayer pores, $\Psi$. Contrarily, if the contact angle $\Theta$ exceeds the re-entrant angle $\Psi$, the liquid forms a convex meniscus shape at the pore entrance, effectively preventing the liquid from filling the pore. ${ }^{29}$ The wetting situation is analogous to liquid infiltration into three-dimensional pore arrays in inverse opal structures, with the notable difference that the inverse monolayer architecture enables tailoring of the involved angles. ${ }^{38-39}$ 

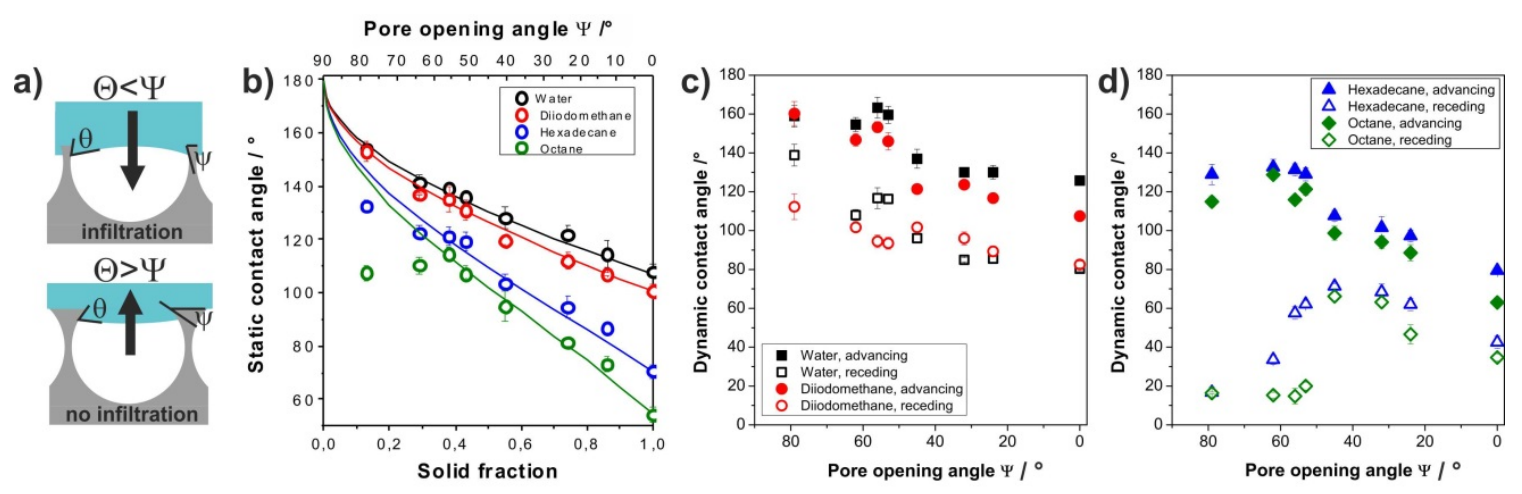

Figure 3. Wetting properties of inverse monolayers with different re-entrant curvature. a) Influence of pore opening angle $\Psi$ on the wettability of a liquids with contact angle $\Theta$. A pore with $\Theta<\Psi$ will be infiltrated by the liquid, leading to a Wenzel wetting state (top). If $\Theta>\Psi$, a convex meniscus shape results that expels the liquid from the pore, forming a Cassie-Baxter wetting state (bottom). b) Static contact angles of various liquids as a function of the solid fraction $\Phi$ and the pore opening angle $\Psi$ of the inverse monolayer structures. The solid line shows the calculated contact angles for a Cassie-Baxter wetting scenario. c,d) Dynamic contact angles of water and diiodomethane (c) and hexadecane and octane (d) as a function of the pore opening angle $\Psi$. A sudden increase in receding contact angles for hexadecane and octane (d) marks the transition between Wenzel- and Cassie wetting.

Figure 3b shows the static contact angle of all test liquids measured on inverse monolayer surfaces with pore opening angles between $79^{\circ}$ and $23^{\circ}$. As shown in Figure 1e and Table 1, the pore opening angle directly relates to the surface coverage of solid material at the plane of the pores; i.e. the higher the pore opening angle, the lower the fraction of solid material. We compare the experimental results with the predicted contact angles by the Cassie-Baxter equation (shown as solid lines) that connects the contact angle on a rough surface $\Theta^{*}$ with the contact angle of a liquid on a flat surface $\Theta$ with the solid fraction $\Phi$ in contact with the liquid. ${ }^{40}$

$$
\cos \Theta^{*}=-1+\Phi(\cos \Theta+1)
$$

The contact angles of water and diiodomethane, both having relatively high surface tensions, closely follow the values predicted for a Cassie-Baxter wetting state with all pore opening angles. The contact angle values for hexadecane and octane only follow the Cassie-Baxter predictions for smaller pore openings. At higher opening angles, the values deviate to lower contact angles, indicating liquidinfiltration into the pores and the formation of a Wenzel wetting state. The transition from Cassie-Baxter to Wenzel wetting becomes even more obvious with the analysis of dynamic wetting conditions. In Figure 
3c,d, we plot the advancing and receding contact angle of the test liquids as a function of the pore opening angle. As expected, water and diiodomethane (Figure 3c) show high receding contact angles on surfaces with any pore opening angle, indicating absence of pore filling and thus decreased pinning of the droplets on the surface. For hexadecane and octane (Figure 3d), a sharp increase in receding contact angles can be observed. At high pore openings, the receding contact angle is very low. With decreasing pore opening angles, the receding contact angle increases strongly and suddenly upon exceeding a critical pore opening angle (approximately $60^{\circ}$ for hexadecane and $50^{\circ}$ for octane). The increase in receding contact angle with smaller pore opening angles marks the transition between Wenzel and Cassie-Baxter wetting. In the former case, low values of the receding contact angles are caused by strong pinning of the liquid in contact with the pore surface. The experimentally observed transition in wetting correlates with the expected values from the contact angles on a flat surface (around $65^{\circ}$ for hexadecane and $50^{\circ}$ for octane; compare to Figure 2). The pore infiltration and thus the macroscopic wetting properties of a liquid can thus be controlled by adjusting the pore opening angle to be below the critical value given by the contact angle of the liquid.

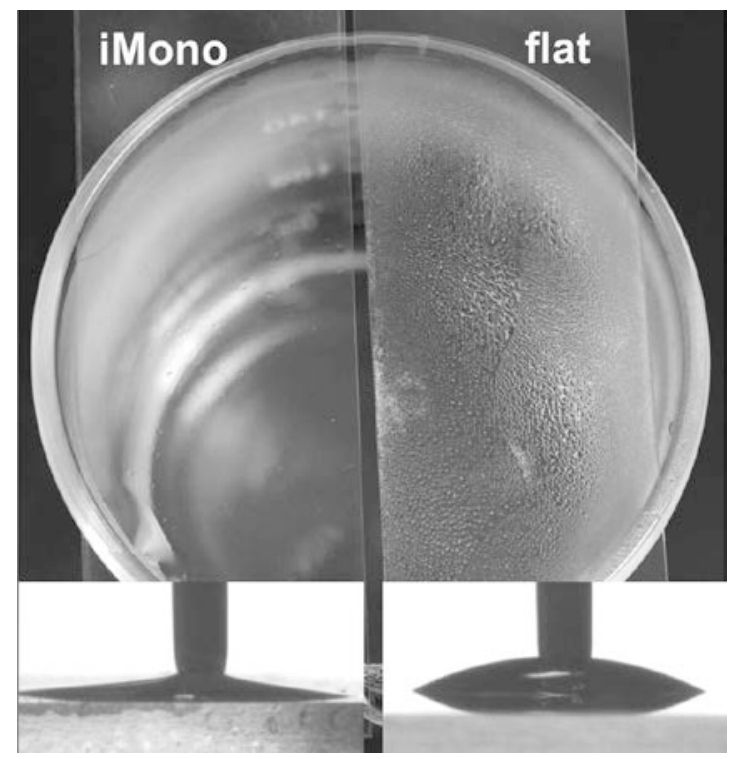

Figure 4. Superhydrophilic and anti-fogging properties of inverse monolayer coatings (labelled “iMono”, prepared from 200nm colloids as templates with pore opening angles of $90^{\circ}$ ) compared to plain glass slides. The top part of the figure shows a photograph of the two samples on a beaker containing hot water. The steam condenses on the surface and reveals the different wetting properties. While a flat water film forms on the inverse monolayer (the substrate remains transparent since the uniform water film does not scatter light), individual condensation droplets are observed on the flat reference, compromising visibility. The inverse monolayer thus shows anti-fogging properties with retained transparency. The lower part 
shows image takes during contact angle measurements. A clear difference in contact angles between the inverse monolayer surface (contact angle approaching $0^{\circ}$ ) and the reference substrate (contact angle approximately $25^{\circ}$ ) can be seen.

While a Cassie-Baxter wetting state enables the design of repellent surfaces or the separation of liquids with different contact angles, the controlled infiltration of a liquid into a pore can be advantageous as well. Compared to a flat surface, Wenzel-type wetting on a porous surface enables the formation of superhydrophilic surfaces, with contact angles approaching $0^{\circ}$ as the liquid is drawn into the surface features. $^{21-22}$ Without additional surface functionalization, the naturally hydrophilic silica surface of the inverse monolayer shows superhydrophilic properties: a water droplet rapidly spreads onto the surface (Figure 4). This property creates anti-fogging surfaces where water droplets condensing onto the surface rapidly spread into a homogeneous thin film, maintaining transparency by prevention of light scattering at individual water droplets (Figure 4). We chose inverse monolayer coatings prepared from smaller colloidal particles $(200 \mathrm{~nm})$ since their small size leads to complete transparency of the nanostructured coating, thus facilitating comparison with the reference glass substrate. Inverse monolayer coatings prepared form larger colloids described above showed similar superhydrophilic behavior. In contrast, condensation on a flat reference surface occurs in small droplets and compromises transparency. Such anti-fogging properties may be of interest in combination with the attractive mechanical properties of inverse monolayers. Due to their closed-cell structure, the surface coating has been shown to be mechanically robust and not easily damaged by abrasion and mechanical contact such as touching or wiping. $^{35}$

Micropatterning of surface structures can be used to spatially tailor the wetting properties of a surface, for example to create patterns of superhydrophilic and superhydrophobic areas. ${ }^{41}$ This precise confinement of aqueous solutions to designated parts of the surface finds application in a wide range of research areas, including multiplexed cell culture and cell isolation without cross-contamination, ${ }^{42-43}$ microfluidic chips with controlled water transport following predefined pathways, ${ }^{44-46}$ highly-sensitive detection of analytes, ${ }^{44,} 47-48$ capillary-driven self-alignment of microscopic objects, ${ }^{49}$ dynamic control of patterning, ${ }^{50}$ or chemical synthesis in confinement; for example to fabricate metal-organic framework microsheets with tailored dimensions. ${ }^{51}$ However, the spatial confinement of low-surface-tension liquids such as organic solvents is substantially more difficult because of their highly wetting behavior that has been shown so far to be prevented only by creating quite complex, lithographically defined surface structures. ${ }^{52-53}$

In Figure 5, we demonstrate the use of micropatterning to locally define the surface functionalization of the inverse monolayer coatings. This enables us to tailor the wetting state of low-surface-tension liquids 
on macro- and microscopic areas with high accuracy and thus confine low-surface-tension liquids to designated areas of the substrate. Using photolithography, we can selectively expose defined surface areas which are subsequently fluorosilanized (indicated by an orange color in Figure 5a), while the parts of the surface blocked by the photoresist layer remain unfunctionalized (indicated as green color in Figure 5a). After photoresist removal, we obtain an inverse monolayer that shows repellent properties only in the designated, fluorosilanized surface regions exposed by the patterning process. Tailoring the re-entrant geometry to enable Cassie-Baxter wetting in the surface-functionalized regions, a low-surface-tension liquid added to the surface will de-wet from the fluorinated areas and be confined to the nonfunctionalized areas. Figure 5b shows the confinement of octane to a macroscopic area. The time-lapsed images show the subsequent drying of octane: no physical barriers are present and the confinement is completely driven by a combination of defined (Cassie-Baxter) wetting states from the re-entrant geometry and the locally compromised surface functionality. Figure 5c shows the high accuracy with which the low-surface-tension liquid is confined to the surface. The wetted areas follow the desired pattern with the resolution of single pores $(1 \mu \mathrm{m}$ diameter in the example). No wicking of octane into undesired parts of the surface is observed.
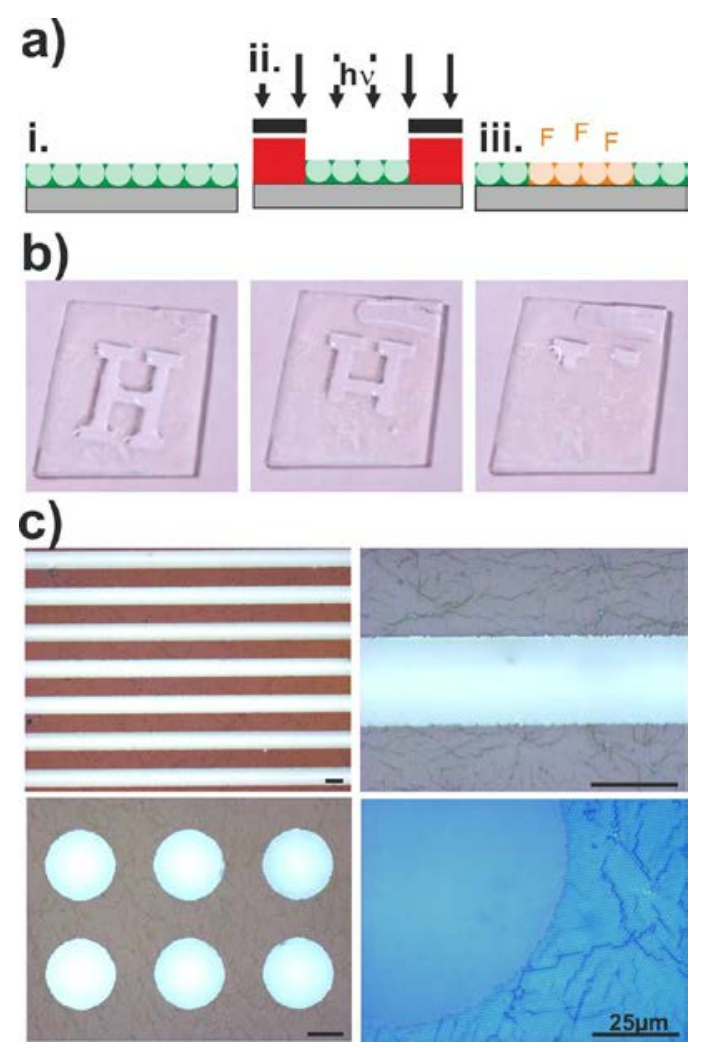

Figure 5. Spatial confinement of low-surface-tension liquids by patterned surface chemistry. a) Schematic illustration of the photolithographic process used to patterning the surface chemistry into repellent, fluorinated surface and wettable, unmodified areas. b) Time-lapsed images demonstrating the spontaneous 
confinement of octane into desired parts of the surface. Upon drying, the pristine surface is regenerated, showing the absence of any physical barriers. c) Microscopy images of micron-scale surface patterns of octane. The close-up images clearly resolve visible, non-wetted inverse monolayer structures and wetted areas. The high precision of the confinement can be seen by the complete absence of leakage of octane into fluorinated areas. All scale bars are $50 \mu \mathrm{m}$ unless stated otherwise.

In conclusion, we show how a simple colloidal templating process can be used to accurately predict and tailor the macroscopic wetting state of liquids with varying surface tensions. We create inverse monolayers by backfilling a two-dimensional colloidal crystal with a silica sol-gel precursor and are able to precisely tailor the opening angle of the individual pores via a variation of the precursor concentration. This pore opening angle is the crucial parameter that characterizes the re-entrant curvature of the porous coating which, in turn, determines the wetting state of the liquid. If the pore opening angle is smaller than the contact angle of the liquid, pore infiltration is prevented, leading to a Cassie-Baxter wetting state. We prepare inverse monolayers with a wide range of pore opening angles and show that the macroscopic wetting properties of liquids with varying surface tensions can be controlled. We further locally pattern the surface functionality via photolithography which allows us to confine low-surface-tension liquids to designated areas of the surface with high accuracy. The ability to tailor wetting properties with high surface tension selectivity in a simple self-assembly process impacts various technologies and applications, from simplified tests of surface tension to separation membranes for low-surface-tension liquids or chemical reactions in spatially confined regions.

\section{Experimental details}

\section{Materials}

All materials were purchased from Sigma Aldrich and used without further purification, except for styrene which was distilled at reduced pressure to remove the inhibitor.

\section{Colloidal synthesis and assembly into monolayers}

Colloids were synthesized by surfactant-free emulsion polymerization with acrylic acid as comonomer as described elsewhere. ${ }^{54}$ Self-assembly was performed using the air/water interface as a template following a protocol from literature. In brief, the colloidal dispersion was diluted with ethanol (50\% by volume) and added to the air/water interface via a hydrophilic glass slide immersed into the water subphase at an angle of approximately $45^{\circ}$. At the three phase contact line, the colloids assemble into a close-packed 
monolayer. Addition was continued until the entire air/water interface was covered by a monolayer. Subsequently, a substrate was immersed into the subphase and withdrawn under a shallow angle to transfer the monolayer.

\section{Inverse monolayer preparation}

Tetraethylorthosilicate, hydrochloric acid $(0.1 \mathrm{~N})$ and ethanol were mixed with ratios specified in Table 1 and stirred for $1 \mathrm{~h}$ at room temperature for prehydrolysis. The solution was diluted with ethanol to control the pore opening (values specified in Table 1) and added onto the monolayer-coated substrate $\left(10 \mu \mathrm{l} / \mathrm{cm}^{2}\right)$. The solution was uniformly spread onto the substrate by tilting and subsequently spincoated at 3000rpm for 30s. Calcination was performed at $500^{\circ} \mathrm{C}$ (heating from $23^{\circ} \mathrm{C}$ to $500^{\circ} \mathrm{C}$ for 5 h, temperature kept constant for $2 \mathrm{~h}$, cooling for $5 \mathrm{~h}$ to $23^{\circ} \mathrm{C}$ ). All samples were characterized by scanning electron microscopy on a Zeiss Ultra SEM.

Surface functionalization was performed with $(1 \mathrm{H}, 1 \mathrm{H}, 2 \mathrm{H}, 2 \mathrm{H}$-tridecafluorooctyl)-trichlorosilane using vapor phase silanization for $24 \mathrm{~h}$ at reduced pressure and room temperature. Prior to silanization, the substrates were cleaned in base piranha (1:1:5 ammonia (25\%):hydrogen peroxide (33\%):ultrapure water) and plasma-treated with oxygen plasma (5min, $100 \mathrm{~W}, 5 \mathrm{sccm} \mathrm{O}_{2}$ flow):

\section{Photolithography}

A positive tone photoresist (S1818, Shipley) was spincoated (4000 rpm, $60 \mathrm{~s}$ ) onto the inverse monolayer substrate and illuminated with UV light as specified by the manufacturer (irradiation dose: $200 \mathrm{~mJ} \mathrm{~cm}^{-2}$ ). Afterwards, the pattern was developed by immersion into a commercial developer solution (Shipley, Developer) for $60 \mathrm{~s}$. The patterned substrate was plasma-treated $\left(\mathrm{O}_{2}\right.$ plasma, $100 \mathrm{~W}$, $5 \mathrm{sccm}$ flow $)$ for $5 \mathrm{~min}$ and then surface-functionalized following the protocol described above. Finally, the resist was removed from the surface (Remover PG 1165, Shipley), leaving the uncovered surface areas without fluorinated surface groups.

\section{Contact angle measurements}

Contact angles were measured at room temperature without control of humidity. Droplets with a volume of $3 \mu \mathrm{l}$ were added to the substrate via a syringe and the droplet profile was analyzed with the software provided by the manufacturer (Krüss $\mathrm{GmbH}$ ). Advancing and receding contact angles were measured by increasing and decreasing the droplet volume while taking screenshots. All values for contact angles given in the main text were averaged over at least 10 different measurements. 


\section{Acknowledgements}

N.V. acknowledges funding of the Deutsche Forschungsgemeinschaft (DFG) through the Cluster of Excellence Engineering of Advanced Materials. S.U, acknowledges the Deutsche Forschungsgemeinschaft (DFG) for financial support.

\section{References}

1 N. Vogel, M. Retsch, C.-A. Fustin, A. del Campo, U. Jonas, Chem. Rev. 2015, 115, 6265-6311.

2 A. Stein, B. E. Wilson, S. G. Rudisill, Chem. Soc. Rev. 2013, 42, 2763-2803.

3 K. R. Phillips, G. T. England, S. Sunny, E. Shirman, T. Shirman, N. Vogel, J. Aizenberg, Chem. Soc. Rev. 2016, DOI: 10.1039/C5CS00533G

$4 \quad$ J. H. Zhang, Y. F. Li, X. M. Zhang, B. Yang, Adv. Mater. 2010, 22, 4249-4269.

$5 \quad$ N. Vogel, C. K. Weiss, K. Landfester, Soft Matter 2012, 8, 4044-4061.

6 T. Kraus, D. Brodoceanu, N. Pazos-Perez, A. Fery, Adv. Funct. Mater. 2013, 23, 4529-4541.

$7 \quad$ M. Kolle, P. M. Salgard-Cunha, M. R. J. Scherer, F. M. Huang, P. Vukusic, S. Mahajan, J. J. Baumberg, U. Steiner, Nat. Nanotechnol. 2010, 5, 511-515.

8 C. Park, K. Koh, U. Jeong, Scientific Reports 2015, 5, 8340.

9 M. Karg, T. A. F. König, M. Retsch, C. Stelling, P. M. Reichstein, T. Honold, M. Thelakkat, A. Fery, Mater. Today 2015, 18, 185-205.

10 J. Bravo, L. Zhai, Z. Wu, R. E. Cohen, M. F. Rubner, Langmuir 2007, 23, 7293-7298.

11 Y. Li, J. Zhang, S. Zhu, H. Dong, F. Jia, Z. Wang, Z. Sun, L. Zhang, Y. Li, H. Li, W. Xu, B. Yang, Adv. Mater. 2009, 21, 4731-4734.

12 L. Xu, J. He, Langmuir 2012, 28, 7512-7518.

13 H.-J. Tsai, Y.-L. Lee, Langmuir 2007, 23, 12687-12692.

14 S. G. Park, S. Y. Lee, S. G. Jang, S. M. Yang, Langmuir 2010, 26, 5295-5299.

$15 \quad$ K. Ellinas, A. Tserepi, E. Gogolides, Langmuir 2011, 27, 3960-3969.

16 J. Wang, Y. Zhang, S. Wang, Y. Song, L. Jiang, Accounts Chem. Res. 2011, 44, 405-415.

17 L. Junyan, W. Li, B. Jingxian, H. Ling, J. Mater. Chem. A 2015, 3, 20134-20144.

18 W. Barthlott, C. Neinhuis, Planta 1997, 202, 1-8.

19 L. Feng, S. Li, Y. Li, H. Li, L. Zhang, J. Zhai, Y. Song, B. Liu, L. Jiang, D. Zhu, Adv. Mater. 2002, 14, 1857-1860.

20 W. Lee, M.-K. Jin, W.-C. Yoo, J.-K. Lee, Langmuir 2004, 20, 7665-7669.

21 X. J. Feng, L. Jiang, Adv. Mater. 2006, 18, 3063-3078.

22 K. Liu, X. Yao, L. Jiang, Chem. Soc. Rev. 2010, 39, 3240-3255.

23 A. B. D. Cassie, S. Baxter, Trans. Faraday Soc. 1944, 40, 0546-0550.

24 T. Darmanin, F. Guittard, J. Mater. Chem. A 2014, 2, 16319-16359.

25 P. Ragesh, V. Anand Ganesh, S. V. Nair, A. S. Nair, J. Mater. Chem. A 2014, 2, 14773-14797.

26 R. N. Wenzel, Ind. Eng.Chem. 1936, 28, 988-994.

27 A. Tuteja, W. Choi, M. L. Ma, J. M. Mabry, S. A. Mazzella, G. C. Rutledge, G. H. McKinley, R. E. Cohen, Science 2007, 318, 1618-1622.

A. Ahuja, J. A. Taylor, V. Lifton, A. A. Sidorenko, T. R. Salamon, E. J. Lobaton, P. Kolodner, T. N. Krupenkin, Langmuir 2008, 24, 9-14.

29 A. Tuteja, W. Choi, J. M. Mabry, G. H. McKinley, R. E. Cohen, Proc. Natl. Acad. Sci. U. S. A. 2008, 105, 18200-18205. H. J. Butt, C. Semprebon, P. Papadopoulos, D. Vollmer, M. Brinkmann, M. Ciccotti, Soft Matter 2013, 9, 418-428.

T. Jiang, Z. Guo, W. Liu, J. Mater. Chem. A 2015, 3, 1811-1827. 
Y. Li, W. P. Cai, B. Q. Cao, G. T. Duan, F. Q. Sun, C. C. Li, L. C. Jia, Nanotechnology 2006, 17, 238-243.

34 Y. Li, C. C. Li, S. O. Cho, G. T. Duan, W. P. Cai, Langmuir 2007, 23, 9802-9807.

35 N. Vogel, R. A. Belisle, B. Hatton, T.-S. Wong, J. Aizenberg, Nat Commun 2013, 4, 2167. DOI:10.1038/ncomms3176

36 S. Akerboom, S. P. Pujari, A. Turak, M. Kamperman, ACS Appl. Mater. Interfaces 2015, 7, 16507-16517.

37 N. Vogel, S. Goerres, K. Landfester, C. K. Weiss, Macromol. Chem. Phys. 2011, 212, 1719-1734.

38 I. B. Burgess, N. Koay, K. P. Raymond, M. Kolle, M. Loncar, J. Aizenberg, ACS Nano 2012, 6, 1427-1437.

39 I. B. Burgess, L. Mishchenko, B. D. Hatton, M. Kolle, M. Lončar, J. Aizenberg, J. Amer. Chem. Soc. 2011, 133, 12430-12432.

40 P.-G. de Gennes, F. Brochard-Wyart, D. Quere, Capillarity and Wetting Phenomena, Springer, New York, 2004.

41 E. Ueda, P. A. Levkin, Adv. Mater. 2013, 25, 1234-1247.

$42 \quad$ F. L. Geyer, E. Ueda, U. Liebel, N. Grau, P. A. Levkin, Angew. Chem.-Int. Edit. 2011, 50, 84248427.

43 H. Li, Q. Yang, G. Li, M. Li, S. Wang, Y. Song, ACS Appl. Mater. Interfaces 2015, 7, 9060-9065.

44 A. W. Martinez, S. T. Phillips, M. J. Butte, G. M. Whitesides, Angew. Chem.-Int. Edit. 2007, 46, 1318-1320.

45 H. Mertaniemi, V. Jokinen, L. Sainiemi, S. Franssila, A. Marmur, O. Ikkala, R. H. A. Ras, Adv. Mater. 2011, 23, 2911-2914.

46 I. You, S. M. Kang, S. Lee, Y. O. Cho, J. B. Kim, S. B. Lee, Y. S. Nam, H. Lee, Angew. Chem.Int. Edit. 2012, 51, 6126-6130.

47 J. Hou, H. Zhang, Q. Yang, M. Li, Y. Song, L. Jiang, Angew. Chem.-Int. Edit. 2014, 53, 57915795.

48 J. Hou, H. Zhang, Q. Yang, M. Li, L. Jiang, Y. Song, Small 2015, 11, 2738-2742.

49 B. Chang, A. Shah, Q. Zhou, R. H. A. Ras, K. Hjort, Scientific Reports 2015, 5, 14966.

50 A. N. Efremov, M. Grunze, P. A. Levkin, Adv. Mater. Int. 2014, 1, 1300075, DOI:10.1002/admi.201300075

51 M. Tsotsalas, H. Maheshwari, S. Schmitt, S. Heißler, W. Feng, P. A. Levkin, Adv. Mater. Int. 2015, DOI:10.1002/admi.201500392

52 S. M. Kang, C. Lee, H. N. Kim, B. J. Lee, J. E. Lee, M. K. Kwak, K. Y. Suh, Adv. Mater. 2013, 25, 5756-5761.

53 V. Liimatainen, V. Sariola, Q. Zhou, Adv. Mater. 2013, 25, 2275-2278.

54 N. Vogel, L. de Viguerie, U. Jonas, C. K. Weiss, K. Landfester, Adv. Funct. Mater. 2011, 21, 3064-3073. 


\section{TOC image}

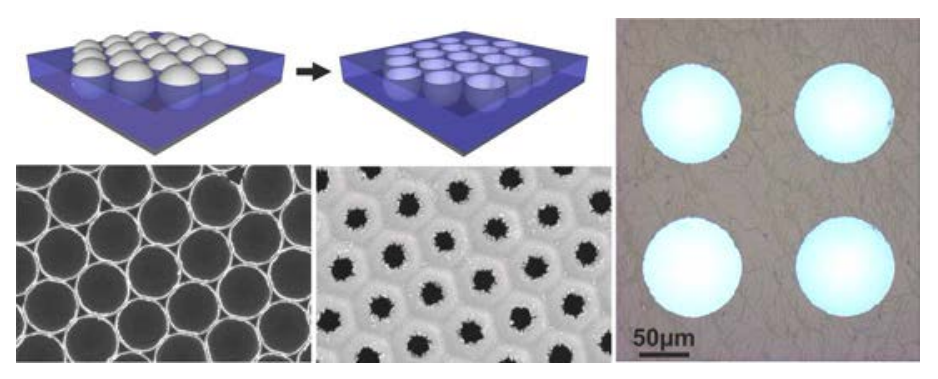

Two-dimensional nanopore arrays with adjustable re-entrant curvature are prepared from colloidal templating and used to pattern low-surface-tension liquids. 